\title{
Continuous size fractionation of magnetic nanoparticles by using simulated moving bed chromatography
}

\author{
Carsten-Rene Arlt ${ }^{1}$, Dominik Brekel ${ }^{1}$, Stefan Neumann $^{2}$, David Rafaja ${ }^{2}$, Matthias Franzreb (ه) ${ }^{1}$ \\ 1 Institute of Functional Interfaces, Karlsruhe Institute of Technology, 76344 Eggenstein-Leopoldshafen, Germany \\ 2 Institute of Materials Science, TU Bergakademie Freiberg, 09599 Freiberg, Germany
}

(C) The Author(s) 2021. This article is published with open access at link.springer.com and journal.hep.com.cn

\begin{abstract}
The size fractionation of magnetic nanoparticles is a technical problem, which until today can only be solved with great effort. Nevertheless, there is an important demand for nanoparticles with sharp size distributions, for example for medical technology or sensor technology. Using magnetic chromatography, we show a promising method for fractionation of magnetic nanoparticles with respect to their size and/or magnetic properties. This was achieved by passing magnetic nanoparticles through a packed bed of fine steel spheres with which they interact magnetically because single domain ferro-/ferrimagnetic nanoparticles show a spontaneous magnetization. Since the strength of this interaction is related to particle size, the principle is suitable for size fractionation. This concept was transferred into a continuous process in this work using a so-called simulated moving bed chromatography. Applying a suspension of magnetic nanoparticles within a size range from 20 to $120 \mathrm{~nm}$, the process showed a separation sharpness of up to 0.52 with recovery rates of $100 \%$. The continuous feed stream of magnetic nanoparticles could be fractionated with a space-time-yield of up to $5 \mathrm{mg} /(\mathrm{L} \cdot \mathrm{min})$. Due to the easy scalability of continuous chromatography, the process is a promising approach for the efficient fractionation of industrially relevant amounts of magnetic nanoparticles.
\end{abstract}

Keywords magnetic chromatography, simulated moving bed chromatography, magnetic nanoparticles, size fractionation

\section{Introduction}

Magnetic nanoparticles (MNPs) are becoming increasingly

Received November 24, 2020; accepted January 14, 2021

E-mail: matthias.franzreb@kit.edu important in many medical and technical disciplines. In the medical field, these include, for example, gene therapy [1] or cancer treatment by targeted drug delivery and magnetic hyperthermia, since the use of spatially focused magnetic fields in combination with functionalized MNPs allows to provide unique specificity to certain therapies [2-6]. Furthermore, a promising application as a contrast agent in MRI diagnoses is apparent [7-9]. On the technical side, MNPs are increasingly used for sensing [10], magnetic bearings [11], waste water treatment [12,13] and separation techniques [14]. An important quality parameter for MNPs is the uniformity of their particle size $[15,16]$. Even if a nominal particle size of the MNPs can be described after the synthesis, there is usually a more or less broad size distribution due to process-related reasons [17-19]. Besides optimizing particle synthesis, the quality of the MNP product line can be improved by subsequent fractionation. However, in the size range of about 20 $200 \mathrm{~nm}$ fractionation processes encounter technical difficulties, which so far have only been solved to a limited extent. This problem is also apparent, for example, in high efficiency particulate air filters, where this size range is known as "most penetrating particle size" [20-22]. The reason for this separation gap is that there is a complex overlapping of forces in this size range. For example, volume forces are particularly important [23] for fractionation processes from the micrometer range onwards, while Brownian motion, diffusion and convection play a major role in the sub-nanometer scale [24]. For these reasons, the high-resolution size fractionation of MNPs continues to be an intensively studied scientific field. In this context, size separation means a selective fractionation of particles with a broad size distribution into two or more fractions, which differ in their mean particle diameter. So far, various processes have been investigated for this purpose, including micro- and ultrafiltration [25,26], acoustic fractionation [23,27], gel electrophoresis [28] and ultracentrifugation $[29,30]$. An established separation process 
for MNPs is the use of so-called high gradient magnetic separators [31,32]. However, the strong magnetic fields and field gradients used in these apparatus result in a practically complete separation of all magnetic particles in the feed stream. Therefore, these separators only have very limited efficiencies when being considered for size fractionations. Other separation processes such as field flow fractionation can achieve reproducible size fractionations, however, they are only suitable for analytical scale $[33,34]$. Another variant we want to emphasize in this work is the usage of liquid chromatography methods for the fractionation of ultrafine particles. Through the use of size exclusion chromatography, it has already been possible to fractionate batches of nanoparticles in a range of 5-50 nm according to their size $[35,36]$. The approach pursued in this work comprises the magnetic interaction of MNPs with a magnetically responsive chromatography matrix. Some studies, such as those by Nomizu, Ohara and Kim et al. [37-39], used magnetochromatographic methods with external magnetic fields for the fractionation of ultrafine particles. However, in a previous work we already observed size fractionation effects for MNPs in a magnetically responsive matrix without the use of an external magnetic field [40]. For this purpose, a chromatography column was filled with a steel matrix, which showed good suitability due to its high saturation magnetization and low remanence. The fractionation effect could be explained by the fact that MNPs, due to their size, only consist of single-domain magnetic systems, which show a spontaneous magnetization [41]. In addition, the intensity of the magnetic interaction grows with increasing particle size, since larger volume forces will be generated. This special fractionation effect offers many advantages, since an upscaling of the system is simplified due to the missing external magnetic field source. Moreover, the process shows a much lower energy consumption than magnetochromatography using electric coils for field generation. A further advantage is the increased durability of the fractionation matrix materials, since the variation in the magnetic field could lead to a remanence of the same and thus to a loss of MNP and a reduction in fractionation performance. These advantages were enhanced in this study by converting the system from a batch mode to a continuous process by applying the principle of simulating moving bed chromatography (SMB). Satzer et al. were successful in using classical size exclusion SMB for the separation of protein loaded silica nanoparticles with a size of about $70 \mathrm{~nm}$ from unbound protein by means of continuous buffer exchange [42]. The method presented here, shows the further development of a chromatographic approach for size fractionation of MNPs up to $200 \mathrm{~nm}$. To the best of our knowledge, we are the first to show a continuous size fractionation process based on a SMB combined with magnetic chromatography, which also features good scalability.

\section{Experimental}

\subsection{Chemicals and reagents}

In this study, two types of nanoparticles were evaluated. Both nanoparticle types contain iron oxides and show superparamagnetic behavior. Both particle types are commercially available and are prepared by precipitation of iron oxide in the presence of dextran. The first type were cluster-like dextran/iron oxide composite particles (nanomag-D-spio, micromod Partikeltechnologie $\mathrm{GmbH}$, Rostock, Germany) having a density of $1.4 \mathrm{~g} / \mathrm{cm}^{3}$. In dynamic light scattering (DLS) measurements of the particle size, this nanomag-D-spio particles showed a $D_{50}$-value of $43 \mathrm{~nm}$ with a span of 1.19 , which is defined as follows [43]:

$$
\text { Span }=\frac{D_{90}-D_{10}}{D_{50}}
$$

The magnetic properties of the particles were determined using an alternating gradient magnetometer (PMC MicroMag 2900, Princeton Measurement Cooperation, Princeton, USA) revealing a saturation magnetization of $6.1 \mathrm{~A} \cdot \mathrm{m}^{2} / \mathrm{kg}$. The analysis of the crystal structure in a transmission electron microscope (TEM) revealed that these particles contain magnetite as the main crystalline phase (cf. Electronic Supplementary Material, ESM). The second type of nanoparticles consisted of superparamagnetic core-shell particles with a maghemite core, surrounded by a dextran shell (synomag, micromod Partikeltechnologie GmbH, Rostock, Germany). The particles have a density of $2.5 \mathrm{~g} / \mathrm{cm}^{3}$, a $D_{50}$-value of $33.6 \mathrm{~nm}$ with a span of 0.79 , and a saturation magnetization of $48 \mathrm{~A} \cdot \mathrm{m}^{2} / \mathrm{kg}$. The particle size distributions of the MNPs were determined using dynamic light scattering with a Zetasizer (Zetasizer Nano ZSP, Malvern Instruments, Malvern, England) having a detection range between $0.1 \mathrm{~nm}$ and $10 \mu \mathrm{m}$. Particle size determination was carried out in triplicates. The original particle size distributions of the two nanoparticle samples can be seen in Fig. 1. TEM showed that the largest particles in the nanomag sample are agglomerates of single-crystalline magnetite nanoparticles (cf. ESM). The concentration of the nanoparticle suspension samples was determined photometrically using a platereader (EnSpire multimode plate reader, Perkin Elmer, Waltham, USA). For this purpose, $100 \mu \mathrm{L}$ of a particle suspension were pipetted into 96-well UV plates and the absorbance at a wavelength of $280 \mathrm{~nm}$ was measured. To convert the raw data, a calibration plot was generated with defined nanoparticle suspensions of 0.002 to $0.1 \mathrm{~g} / \mathrm{L}$, which showed a reliable linear correlation. Any samples with a higher concentration were diluted accordingly with Tris buffer.

The borosilicate glass chromatography columns (Diba Industries Inc., Danbury, Connecticut) used in this work 
had an inner diameter of $6.6 \mathrm{~mm}$ and formed a chromatography bed length of $120 \mathrm{~mm}$. A PTFE frit each at the beginning and end of the glass column with a pore size of $5 \mu \mathrm{m}$ served as a filter to retain the matrix material and exclude larger impurities. As stationary phase within the magnetic chromatography columns a stainlesssteel powder, TruForm 174 of the company Praxair Surface Technologies, Ratingen, Germany fabricated for 3D selective laser melting was used. The particles consist of a chromium rich $(12.5 \%(\mathrm{w} / \mathrm{w}))$ alloy with small amounts of carbon, silicon and manganese. Their particle size distribution ranges from 5 to $50 \mu \mathrm{m}$ with a $D_{50}$ value of $31 \mu \mathrm{m}$. The particles show a high saturation magnetization of $150 \mathrm{~A} \cdot \mathrm{m}^{2} / \mathrm{kg}$, a very small remanence of $95 \mathrm{~mA} \cdot \mathrm{m}^{2} / \mathrm{kg}$ and a coercivity of $160 \mathrm{~A} / \mathrm{m}$.

\subsection{Experimental setup}

For single column experiments, a Fast Protein Liquid Chromatography system (Äkta purifier, GE Healthcare, Buckinghamshire, England) equipped with polyether ether ketone tubings with an inner diameter of $0.25 \mathrm{~mm}$ was used. In the experiments, the injected sample was pumped through the column at a constant flow rate of $4 \mathrm{~mL} / \mathrm{min}$. The effluent of the column was constantly analyzed by two flow through measuring cells, registering ultraviolet (UV) intensity at $280 \mathrm{~nm}$ and conductivity. For further analysis, a fraction collector divided the effluent into samples with a volume of $0.25 \mathrm{~mL}$. The sample volume injected was $500 \mu \mathrm{L}$ with a nanoparticle concentration of $0.25 \mathrm{mg} / \mathrm{mL}$ in each experiment. In order to guarantee a stable dispersion of the nanoparticle mixture, a buffer system had to be found in which both nanoparticle types did not show agglomeration. A dilute Tris buffer $(1 \mathrm{mmol} / \mathrm{L})$ having a $\mathrm{pH}$ value of 9.5 fulfilled this condition and was used as mobile phase being pumped through the column in all experiments. After each experimental run, the column was thoroughly flushed with buffer in order to remove any residual material.

The experiments for the continuous size fractionation of the nanoparticles were executed with an AZURA Lab simulated moving bed (SMB) system (Knauer Wissenschaftliche Geräte GmbH, Berlin, Germany). A SMB approximates a continuous counter-current operation mode of a chromatographic system, in which the stationary phase moves in opposite direction to the flow direction of the mobile phase. The approximation is achieved by the use of four or more columns and a cyclic interchange of the positions of feed and eluent inlet as well as raffinate and extract outlet by switching valves. If the flow rates of the mentioned in- and outflows as well as the cycle time for switching are chosen correctly, the SMB system can achieve a continuous fractionation of the nanoparticle suspension in the feed flow into two effluent fractions, resulting in a bimodal fractionation. In the course of the separation, the species showing stronger interactions with

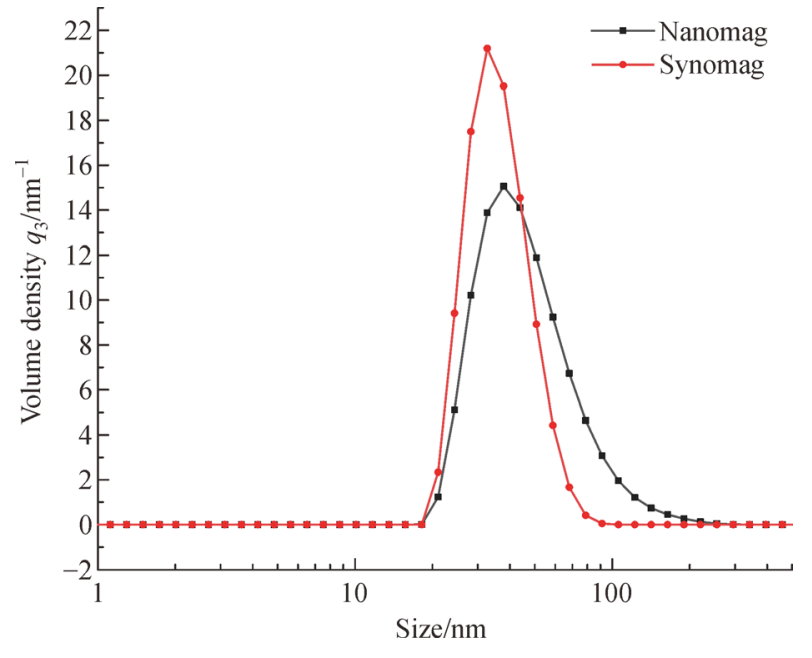

Fig. 1 Dynamic light scattering - size analyses of nanoparticle suspensions containing synomag respectively nanomag nanoparticles. The respective volume density distribution share $q_{3}$ of the nanoparticles was plotted against their hydrodynamic diameter.

the stationary phase will end up in the extract and the species showing weaker interaction with the stationary phase will end up in the raffinate. Due to the growing particle-matrix interaction with increasing particle size, the coarse material is contained in the extract and the fine material in the raffinate respectively. Polyether ether ketone tubings with an inner diameter of $0.75 \mathrm{~mm}$ were used for the connection of the columns and the valves. The flow is controlled by three piston pumps within the loop system and a feed pump which supplies the particle suspension into the system. The flow direction is controlled by seven multi-position valves and by eight check valves. This arrangement would allow the integration of up to eight columns in the system, however, all SMB experiments were conducted by the use of only four columns, one for each zone shown in Fig. 2. By the help of the four pumps, the flow within each zone could be adjusted independently. The online analysis of the extract and the raffinate was performed using two UV cells at $280 \mathrm{~nm}$. Because of the periodically fluctuating effluent concentrations extract and raffinate samples were pooled over several complete switching cycles for further analyses.

The separation efficiency curve $T(\xi)$ is defined by the mass fraction of particles with a certain characteristic $\xi$, which represents in this work the particle size [36]:

$$
T(\xi)=\frac{m_{\text {coarse }}(\xi)}{m_{\text {feed }}(\xi)}=\frac{m_{\text {coarse }}(\xi)}{m_{\text {coarse }}(\xi)+m_{\text {fines }}(\xi)},
$$

with the respective mass flows $m_{\text {coarse }}$ from the extract and $m_{\text {fines }}$ from the raffinate. This curve can be used to make a quality statement about the separation properties of the system. Knowing the separation efficiency curve, the separation sharpness can be defined by: 


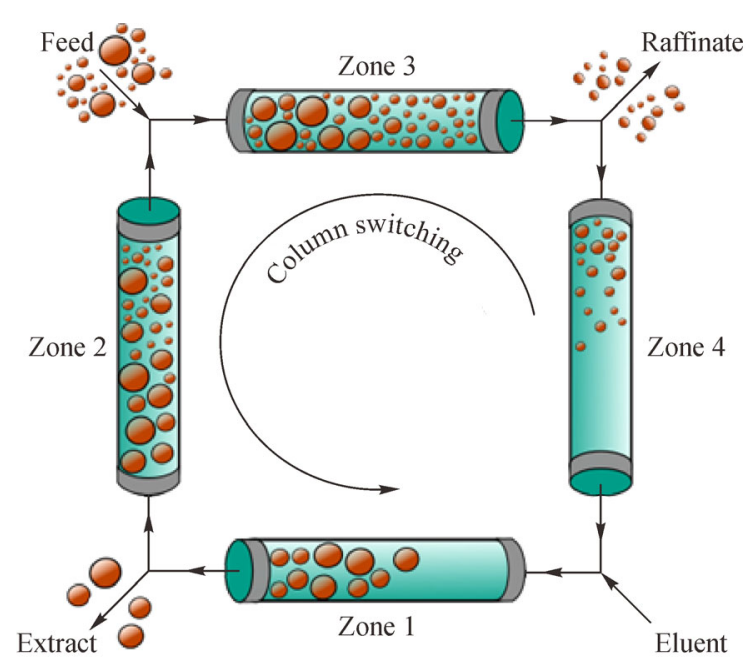

Fig. 2 Scheme of a SMB size fractionation process with a fourcolumn configuration.

$$
k=\frac{T_{25}}{T_{75}},
$$

with $T_{25}$ and $T_{75}$ being the particle sizes at which the separation efficiency curve $T(\xi)$ reaches values of 0.25 and 0.75 , respectively [36].

In order to function properly, SMB chromatography requires carefully selected flow rates in the individual zones. These flow rates are determined from single column experiments. An essential factor for the design of an SMB process is the so-called Henry coefficients $H_{i}$ of the substances to be separated. These coefficients can be determined from the retention times of the single-column tests of the individual components:

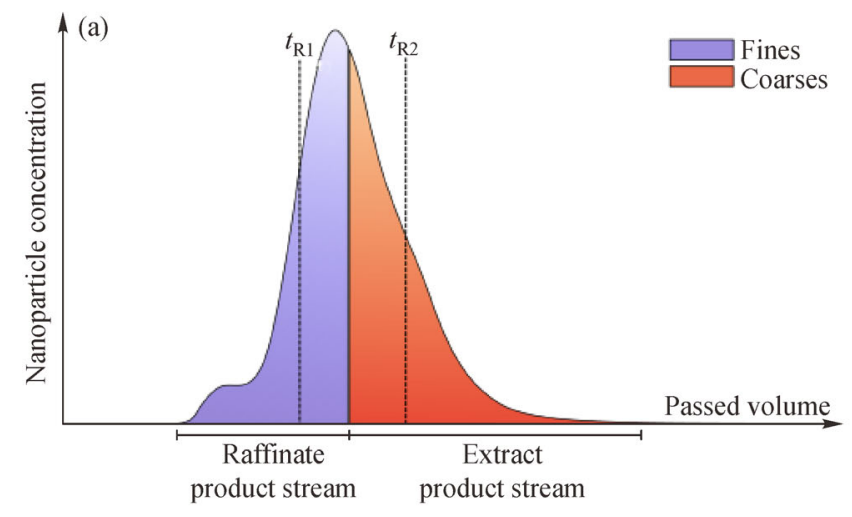

$$
H_{i}=\left(\frac{t_{\mathrm{R} i}}{t_{0}}-1\right) \times \frac{\varepsilon_{\mathrm{t}}}{1-\varepsilon_{\mathrm{t}}} .
$$

With $t_{0}$ as the column void time and $t_{\mathrm{R} i}$ as the retention time of a separation component. However, this classical determination of the necessary parameters is not applicable to the size fractionation shown here. Due to its bimodal separation principle, in most applications SMB is used for the separation of two defined substances. However, a nanoparticle suspension having a broad particle size distribution represents an almost infinite number of different species. In this case, a bimodal separation can be defined by introducing a suitable retention time or retention volume dividing the chromatogram of a corresponding single column experiments into a coarse and a fine fraction, which is visualized in Fig. 3.

A virtual division of the chromatogram is illustrated in Fig. 3(a). This division was now used to determine the necessary Henry coefficients. For this purpose, the respective peak section was assumed to be a single species. The passed volume of each section, where half of the UV-area was eluted, was thus assumed to be the retention time. The division between the sections was chosen in such a way that it halves the total peak area in order to achieve approximately equal mass flows of coarse and fine material. A visualization of this assumption can be taken from Fig. 3(b). For each of the two sections of the peak, a characteristic time can be defined as the retention time of the respective peak area center of the section. $t_{\mathrm{R} 1}$ of the peak area center of the first section corresponds with the time when one quarter of the total particle mass passed the column effluent. Accordingly, $t_{\mathrm{R} 2}$, the retention time of the peak area center of the second section, corresponds to three quarters of the total particle mass:

Fig. 3 Visualization of the determination of characteristic retention times of the coarses and fines fractions. (a) Chromatogram of a single column experiment when injecting a pulse of a nanoparticle suspension having a broad size distribution. Because of the magnetic interaction the retention time of the different particles is size dependent, resulting in a broad peak with smaller particles leaving the column earlier than larger ones. Thus, a first section of the peak can be defined as fines, whereas the second section is defined as coarses. The locations of the respective peak area centers of the two sections define the characteristic retention volumes as well as the corresponding characteristic retention times. From these the respective Henry coefficients can be determined. (b) Visualization of coarse and fine material size distribution as a bimodal separation experiment. 


$$
\frac{1}{4}=\frac{\int_{0}^{t_{\mathrm{R} 1}} c(t) \mathrm{d} t \cdot Q}{M_{\text {total }}} \quad \frac{3}{4}=\frac{\int_{0}^{t_{\mathrm{R} 2}} c(t) \mathrm{d} t \cdot Q}{M_{\text {total }}} .
$$

The time dependent particle concentration is represented by $c(\mathrm{t})$, the single column flow rate by $Q$ and the total particle mass by $M_{\text {total }}$. Knowing the characteristic retention times, the corresponding Henry coefficients for coarses and fines can be calculated according to Eq. (4). For the SMB process, a coordinated selection of the individual flow rates in the four zones illustrated in Fig. 2 is necessary. The flow rates can be defined in a dimensionless form as flow rate ratios $m_{i}$ as follows [44]:

$$
m_{i}=\frac{Q_{i} \times t_{\mathrm{s}}-V_{\mathrm{c}} \times \varepsilon_{\mathrm{t}}}{V_{\mathrm{c}} \times\left(1-\varepsilon_{\mathrm{t}}\right)} .
$$

Here $Q_{i}$ is the flow rate in the respective zone, $V_{\mathrm{c}}$ is the column volume, $t_{\mathrm{s}}$ is the cyclic switching time of the system and $\varepsilon_{\mathrm{t}}$ is the column porosity.

Based on the determined Henry coefficients and the flow rate ratios, the process can now be designed to achieve a stable SMB process with the following conditions according to the triangle method [44,45]:

$$
H_{2} \leqslant m_{2} \leqslant H_{1} ; H_{2} \leqslant m_{3} \leqslant H_{1} ; m_{4} \leqslant H_{2} ; m_{1} \geqslant H_{1} \text {. }
$$

\section{Results and discussion}

\subsection{Single column experiments}

Based on the experience gained in preliminary experiments with smaller columns [40], single column experiments with the new, larger separation columns (5.8 times scale-up in volume) were performed and analyzed. As mentioned in the introduction, the separation mechanism is based on the spontaneous magnetization of the nanoparticles having only a single magnetic domain system. Since the magnetic force is a volume force, the strength of this magnetization depends on the particle size. Consequently, larger particles show a greater magnetic interaction with the steel matrix than smaller ones. If nanoparticle approach the easily magnetizable steel matrix in a distance less than approx. two times their diameter, an attractive magnetic interaction occurs. Since the magnetic force is a volume force, it grows rapidly with increasing particle size. This leads to an only minor retention of small particles and therefore these particle types tend to leave the separation column earlier. As a result, smaller characteristic particle sizes are observed in the first parts of the peak in the chromatogram. In contrast, larger particles encounter a stronger retention and they are generally found at the end of the peak, since the stronger interaction results in a longer residence time in the column. This effect was confirmed with the scaled-up separation matrix. The resulting chromatograms for the tested particle types nanomag and synomag and the results of the DLS measurements of the individual peak sections are shown in Fig. 4. The single-crystalline nature of the nanoparticles was confirmed by TEM (cf. ESM).

Based on the DLS results of the single column experiments, the respective mass median diameter $\left(D_{50}\right)$ of the fractions could be determined assuming a constant particle density. As expected, the average particle size increases in the peak course of the fractionation experiment. In case of the synomag particles the $D_{50}$ increased from $24 \mathrm{~nm}$ in fraction 1 up to $37 \mathrm{~nm}$ in fraction 10. In case of the nanomag particles the difference was even more pronounced, ranging from $32.5 \mathrm{~nm}$ in fraction 1 to $59 \mathrm{~nm}$ in fractions 9 and 10. From the chromatogram peaks, the Henry coefficients could be determined as described in the methods section (see Eqs. (4) and (5)). The respective Henry coefficients are listed in Table 1.

\subsection{Simulated moving bed experiments}

Using the parameters determined in 3.1, the necessary basic operation parameters for continuous multi-column chromatography could now be determined. In the case of the synomag nanoparticles at a feed concentration of $0.25 \mathrm{mg} / \mathrm{mL}$, the continuous mode of operation showed an overloading of the column matrix, which is followed by an unsteady UV-signal and too large mass flows in the raffinate, preventing a reliable fractionation. For this reason, the feed concentration was reduced to $0.05 \mathrm{mg} / \mathrm{mL}$. In the case of the nanomag particles, no breakthrough effects could be observed even at a feed concentration of $0.25 \mathrm{mg} / \mathrm{mL}$, therefore the concentration was maintained. Due to the cyclical principle of an SMB process, an increasing intensity of the extract and raffinate signal can be observed at the beginning of continuous operation. After an operating time of $20 \mathrm{~min}$, the stabilization of the UV signal showed the achievement of a cyclic steady state. This steady state was chosen as a reference point for the continuous separation process. A balance of the feed, extract and raffinate streams based on the photometric measurements of the nanoparticle concentrations showed a recovery rate of the nanoparticles of $100 \%$. This value could be determined over the entire cyclic steady state. The online recording of the UV-signals also allowed determining the mass fraction of the feed stream, which ends up in the extract and raffinate streams, respectively. These fractions are listed in Table 2 . These values were confirmed in photometric offline analyses.

The results show that the assumption of an even distribution of the particle mass into a coarse and fine material stream has been achieved in good approximation. However, it should be noted that the fractionation process results in a dilution of the nanoparticle suspensions with the mobile phase. Based on the DLS measurement, an analysis of the size distribution of raffinate and extract was 

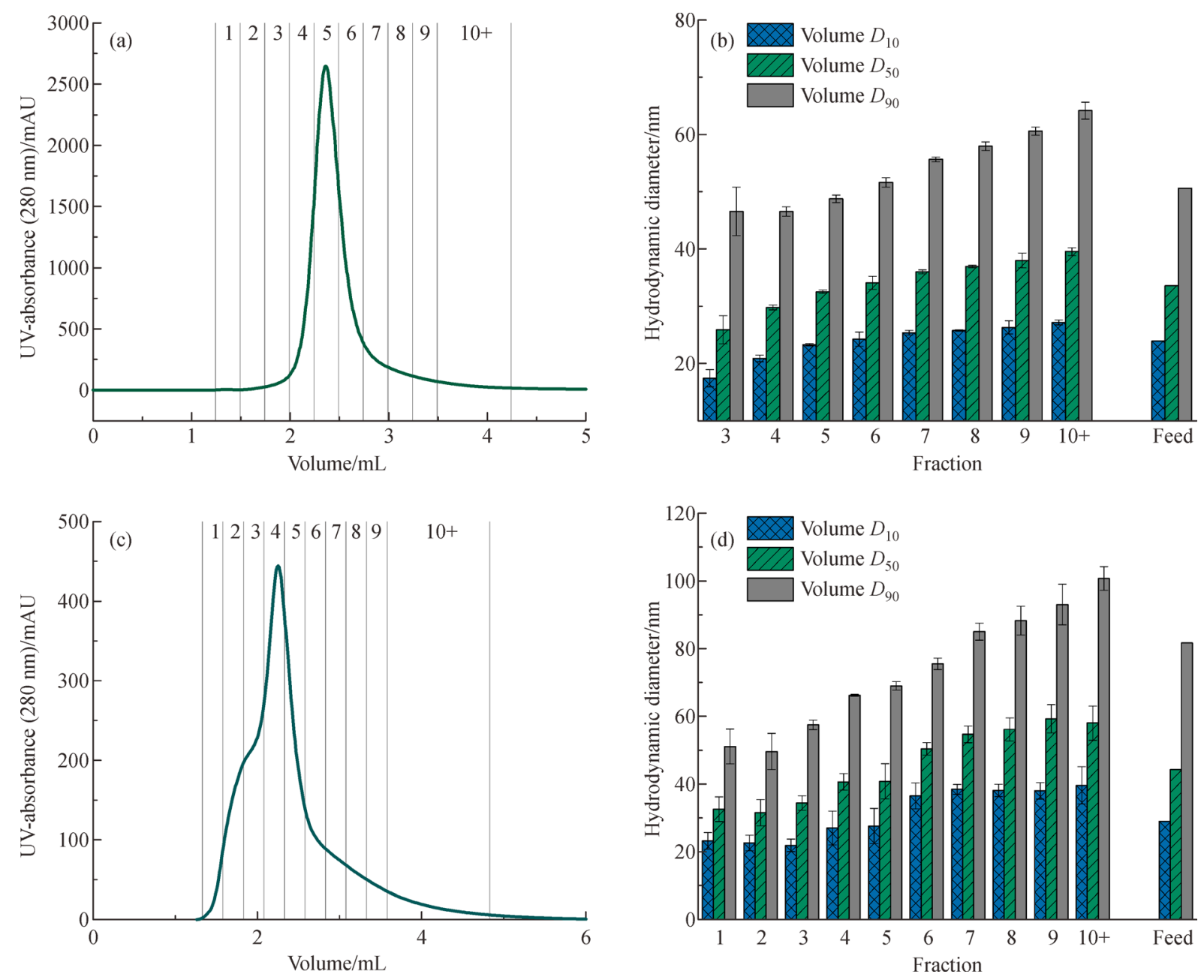

Fig. 4 Chromatograms of the magnetic chromatography experiments for size fractionation of the nanoparticle suspensions and the corresponding DLS analysis results. In the experiments, a sample volume of $500 \mu \mathrm{L}$ containing a nanoparticle suspension of $0.25 \mathrm{mg} / \mathrm{mL}$ was injected into a constant buffer feed stream of $4 \mathrm{~mL} / \mathrm{min}$. The DLS results of the collected effluent samples show the mass median diameter $\left(D_{50}\right)$ and the break points of the largest and smallest $10 \%$ of the nanoparticle species $\left(D_{10}+D_{90}\right)$. (a,b) synomag particles; $(\mathrm{c}, \mathrm{d})$ nanomag particles.

Table 1 Calculated Henry parameters from single column experiments for the determination of the SMB running parameters

\begin{tabular}{lcc}
\hline Nanoparticle sample & Fines Henry coefficient $H_{1}$ & Coarse Henry coefficient $H_{2}$ \\
\hline Synomag & 0.110 & 0.51 \\
Nanomag & 0.105 & 0.6 \\
\hline
\end{tabular}

Table 2 Photometrically determined mass fractions of the product streams of the SMB experiments

\begin{tabular}{lcc}
\hline Nanoparticle sample & Mass fraction of fines (raffinate)/\% & Mass fraction coarse material (extract) $/ \%$ \\
\hline Synomag & 43.3 & 56.6 \\
Nanomag & 47.8 & 52.2 \\
\hline
\end{tabular}

performed. The results of the SMB processes of both nanoparticle types are shown in Fig. 5.

Based on the results shown in Fig. 5, it can be stated that for both nanoparticle types a clear fractionation into coarse and fine particles could be achieved. For example, while the synomag particles in the raffinate show a $15 \%$ smaller average diameter if compared to the average diameter of the particles in the feed, the average particle size in the extract is grown by $8 \%$. In the case of nanomag particles, this difference is even more pronounced with a size 

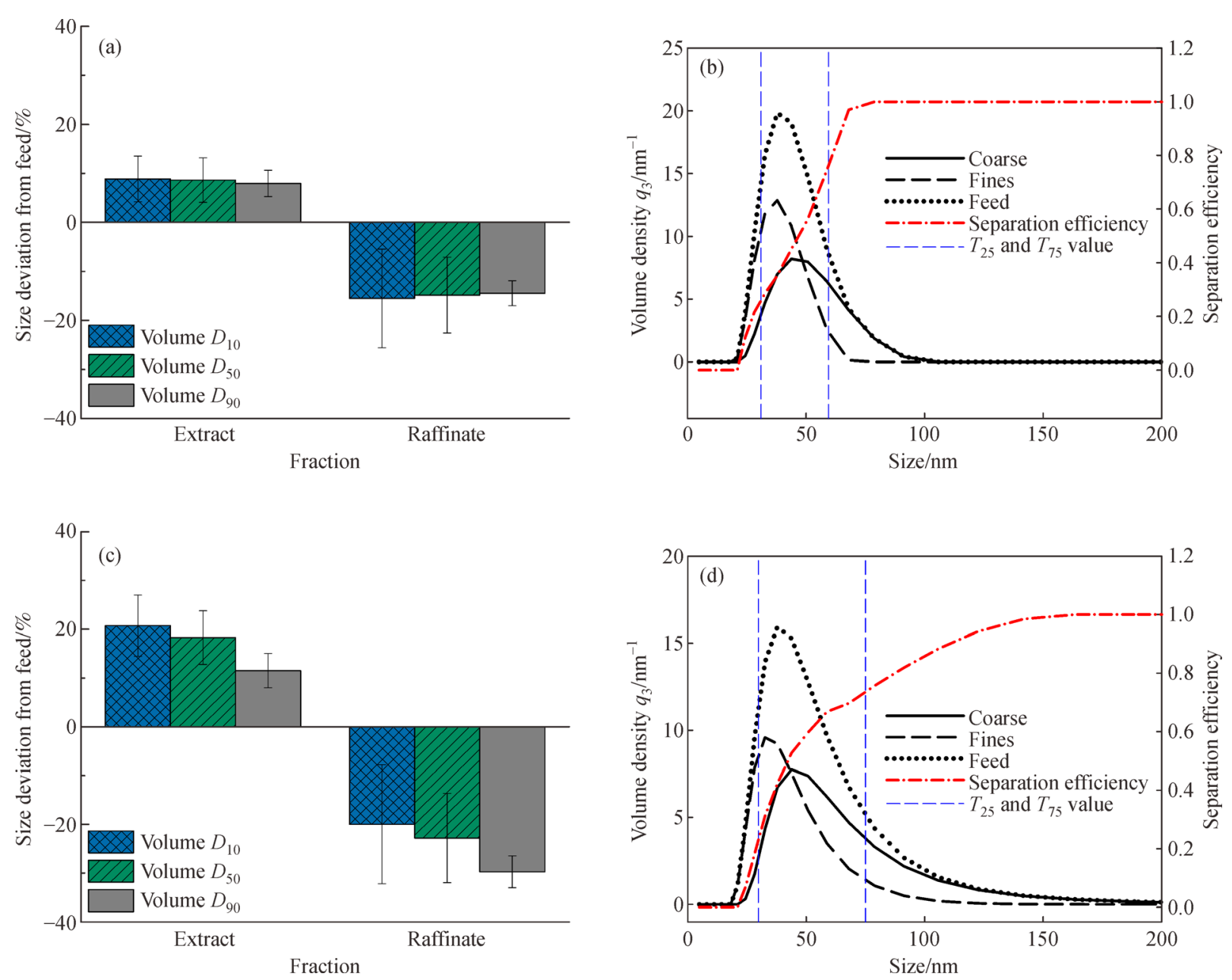

Fig. 5 Results of the size distribution analyses of the product streams resulting from continuous size fractionation experiments of suspensions of synomag $(\mathrm{a}, \mathrm{b})$ and nanomag $(\mathrm{c}, \mathrm{d})$ nanoparticles. The SMB experiments were run in duplicates. DLS analyses were performed to measure the mass median diameter $\left(D_{50}\right)$ and the break points of the largest and smallest $10 \%$ of the nanoparticle species $\left(D_{10}+D_{90}\right)$. (a) and (c) show the relative differences of the particles sizes in the extract and raffinate when compared with the particle sizes in the feed. (b) and (d) show the volume density $\left(q_{3}\right)$ distribution of the feed as well as the coarse and fines fractions together with the resulting separation efficiency curve (red). The blue lines indicate the particle sizes at which the separation efficiency reaches $25 \%$ and $75 \%$.

increase of $18 \%$ in coarse material and a reduction of $22 \%$ in fine material. However, the larger difference may also result from the larger initial size distribution of the nanomag nanoparticles (see Fig. 1). The observed differences are also subject to rather large fluctuations, recognizable in the large standard deviations of up to $9 \%$ in the case of the $D_{50}$ median. Especially in the initial phase of the continuous process these deviations and the errors of the DLS measurements can be amplified due to low particle concentrations [46]. Furthermore, the feed as a reference variable also showed fluctuations, which further amplified the mutual percentage deviations of different runs.

Nevertheless, looking at the separation efficiency curves, the successful fractionations of the two nanoparticle types can be confirmed again. Here too, clear differences in the size distribution between coarse (extract stream) and fine (raffinate stream) particles can be seen. To investigate a change in particle morphology, the coarse and fines fractions were pooled and again analyzed by DLS. This resulted in a size distribution that matched that of the feed. This shows that the particle collective is not changed by the process but simply divided into two fractions. Based on the $T_{25}$ and $T_{75}$ values, the separation sharpness values were calculated. These values were 0.4 in the case of nanomag particles and 0.52 in the case of synomag particles. Thus, both processes are to be acknowledged as technical separations. Compared to the previous results [40], the separation efficiency without an external magnetic field could be increased by using larger columns and consequently larger residence times. Despite the more distinct differences in their average particle size in the coarse and fine fractions, the fractionation using nanomag particles shows a lower separation efficiency than the 
fractionation using synomag particles. This is due to a strong decrease in the slope of the separation curve starting at a hydrodynamic diameter of $50 \mathrm{~nm}$, caused by a more pronounced tailing of the fines fraction towards larger particle species. This could indicate that in this size range a separation mechanism, which is based only on spontaneous magnetization, loses efficiency. This effect is probably enhanced by the lower magnetizability of the nanomag particle type. To further increase the separation efficiency of the continuous process, the application of an external magnetic field, would be a potential approach.

\section{Conclusions}

Starting from size fractionation of nanoparticles using single column magnetic chromatography, a successful transformation of this novel process into a continuous process could be achieved. Furthermore, a successful upscaling could be performed using larger separation columns. Two nanoparticle suspensions were fractionated into a coarses and fines fraction using a modified SMB process. The required operation parameters of the complex SMB system could be extracted from single column experiments according to the established triangle method. In the SMB experiments, separation sharpness values of 0.52 and 0.4 could be achieved. These values indicate that the developed nanoparticle fractionation based on a modified SMB principle achieves useful separations of technical grade. A well-known advantage of liquid chromatographic methods is their easy scalability. With a space-time yield of up to $5 \mathrm{mg} /(\mathrm{L} \cdot \mathrm{min})$, this process is an interesting option, especially if compared to classical ultracentrifugation processes, which require a high energy input [47]. In direct comparison to such a method, the exemplary space-time yield of ultracentrifugation is higher, achieving a value of $8.8 \mathrm{mg} /(\mathrm{L} \cdot \mathrm{min})$. However, the applied ultracentrifugation process was not continuous with regard to the discharge of coarse particles. Therefore, the effective space-time yield will be reduced due to downtimes of the device. Furthermore, in the ultracentrifugation process coarser particle goods were fractionated. The separation mechanism in this work is based on the spontaneous magnetization of single-domain MNPs. Therefore, the application of an external magnetic field could enhance the magnetic forces and further increase the separation efficiencies, as shown in case of single column experiments in our previous work. Consequently, largescale continuous fractionation of nanoparticles by the developed magnetic SMB is thus a promising approach for a cost-efficient quality improvement of MNPs for, e.g., medical use or protective measures against counterfeiting of products.

Acknowledgements The authors acknowledge financial support by the Deutsche Forschungsgemeinschaft (DFG) within the priority program SPP
2045 (Project C11, Grant-Nr. FR 2131/6-1 and Project Z1, Grant-Nr. RA1050/25-1). Furthermore, we thank Mrs. A. Leuteritz for preparing the TEM specimens and the company micromod for the contribution of experimental material.

Electronic Supplementary Material Supplementary material is available in the online version of this article at https://dx.doi.org/10.1007/s11705-0212040-3 and is accessible for authorized users.

Funding note: Open Access funding enabled and organized by Projekt DEAL.

Open Access This article is licensed under a Creative Commons Attribution 4.0 International License, which permits use, sharing, adaptation, distribution and reproduction in any medium or format, as long as you give appropriate credit to the original author(s) and the source, provide a link to the Creative Commons licence, and indicate if changes were made. The images or other third party material in this article are included in the article's Creative Commons licence, unless indicated otherwise in a credit line to the material. If material is not included in the article's Creative Commons licence and your intended use is not permitted by statutory regulation or exceeds the permitted use, you will need to obtain permission directly from the copyright holder. To view a copy of this licence, visit http://creativecommons.org/licenses/by/4.0/.

\section{References}

1. Majidi S, Zeinali S F, Samiei M, Milani M, Abbasi E, Dadashzadeh $\mathrm{K}$, Akbarzadeh A. Magnetic nanoparticles: applications in gene delivery and gene therapy. Artificial Cells, Nanomedicine, and Biotechnology, 2016, 44(4): 1186-1193

2. Hedayatnasab Z, Abnisa F, Daud W. Review on magnetic nanoparticles for magnetic nanofluid hyperthermia application. Materials \& Design, 2017, 123: 174-196

3. Mohammed L, Gomaa H G, Ragab D, Zhu J. Magnetic nanoparticles for environmental and biomedical applications: a review. Particuology, 2017, 30: 1-14

4. Rao L, Bu L L, Meng Q F, Cai B, Deng W W, Li A, Li K, Guo S S, Zhang W F, Liu W, Sun Z J, Zhao X Z. Antitumor plateletmimicking magnetic nanoparticles. Advanced Functional Materials, 2017, 27(9): 1604774

5. Cardoso V F, Francesko A, Ribeiro C, Bañobre L M, Martins P, Lanceros M S. Advances in magnetic nanoparticles for biomedical applications. Advanced Healthcare Materials, 2018, 7(5): 1700845

6. Zhang H, Liu X L, Zhang Y F, Gao F, Li G L, He Y, Peng M L, Fan H M. Magnetic nanoparticles based cancer therapy: current status and applications. Science China. Life Sciences, 2018, 61(4): 400414

7. Kudr J, Haddad Y, Richtera L, Heger Z, Cernak M, Adam V, Zitka O. Magnetic nanoparticles: from design and synthesis to real world applications. Nanomaterials (Basel, Switzerland), 2017, 7(9): 243

8. Demin A M, Pershina A G, Minin A S, Mekhaev A V, Ivanov V V, Lezhava S P, Zakharova A A, Byzov I V, Uimin M A, Krasnov V P, Ogorodova L M. PMIDA-modified $\mathrm{Fe}_{3} \mathrm{O}_{4}$ magnetic nanoparticles: synthesis and application for liver MRI. Langmuir, 2018, 34(11): 3449-3458

9. Arsalani S, Guidelli E J, Silveira M A, Salmon C E G, Araujo J, Bruno A C, Baffa O. Magnetic $\mathrm{Fe}_{3} \mathrm{O}_{4}$ nanoparticles coated by 
natural rubber latex as MRI contrast agent. Journal of Magnetism and Magnetic Materials, 2019, 475: 458-464

10. Gloag L, Mehdipour M, Chen D, Tilley R D, Gooding J J. Advances in the application of magnetic nanoparticles for sensing. Advanced Materials, 2019, 31(48): 1904385

11. Scherer C, Figueiredo Neto A M. Ferrofluids: properties and applications. Brazilian Journal of Physics, 2005, 35(3a): 718-727

12. Bao Y, Wen T, Samia A C S, Khandhar A, Krishnan K M. Magnetic nanoparticles: material engineering and emerging applications in lithography and biomedicine. Journal of Materials Science, 2016, 51 (1): 513-553

13. Ali I, Peng C, Naz I, Khan Z M, Sultan M, Islam T, Abbasi I A. Phytogenic magnetic nanoparticles for wastewater treatment: a review. RSC Advances, 2017, 7(64): 40158-40178

14. Simonsen G, Strand M, Øye G. Potential applications of magnetic nanoparticles within separation in the petroleum industry. Journal of Petroleum Science Engineering, 2018, 165: 488-495

15. Guo X, Wu Z, Li W, Wang Z, Li Q, Kong F, Zhang H, Zhu X, Du Y $\mathrm{P}$, Jin Y, Du Y, You J. Appropriate size of magnetic nanoparticles for various bioapplications in cancer diagnostics and therapy. ACS Applied Materials \& Interfaces, 2016, 8(5): 3092-3106

16. Zhang S, Wu L, Cao J, Wang K, Ge Y, Ma W, Qi X, Shen S. Effect of magnetic nanoparticles size on rheumatoid arthritis targeting and photothermal therapy. Colloids and Surfaces. B, Biointerfaces, 2018, 170: 224-232

17. Baldi G, Bonacchi D, Innocenti C, Lorenzi G, Sangregorio C. Cobalt ferrite nanoparticles: the control of the particle size and surface state and their effects on magnetic properties. Journal of Magnetism and Magnetic Materials, 2007, 311(1): 10-16

18. Ludwig F, Balceris C, Viereck T, Posth O, Steinhoff U, Gavilan H, Costo R, Zeng L, Olsson E, Jonasson C, Johansson C. Size analysis of single-core magnetic nanoparticles. Journal of Magnetism and Magnetic Materials, 2017, 427: 19-24

19. Hara S, Aisu J, Kato M, Aono T, Sugawa K, Takase K, Otsuki J, Shimizu S, Ikake H. One-pot synthesis of monodisperse $\mathrm{CoFe}_{2} \mathrm{O}_{4} @ \mathrm{Ag}$ core-shell nanoparticles and their characterization. Nanoscale Research Letters, 2018, 13(1): 176

20. Lee K W, Liu B Y H. On the minimum efficiency and the most penetrating particle size for fibrous filters. Journal of the Air Pollution Control Association, 1980, 30(4): 377-381

21. da Roza R A. Particle size for greatest penetration of HEPA filters and their true efficiency. Technical Report. 1982

22. Bulejko P, Krištof O, Dohnal M, Svěrák T. Fine/ultrafine particle air filtration and aerosol loading of hollow-fiber membranes: a comparison of mathematical models for the most penetrating particle size and dimensionless permeability with experimental data. Journal of Membrane Science, 2019, 592: 117393

23. Sandmann K, Fritsching U. Selektive partikelklassierung in ultraschallangeregten aerosolen. Chemieingenieurtechnik (Weinheim), 2020, 92(5): 635-642

24. Kowalczyk B, Lagzi I, Grzybowski B A. Nanoseparations: strategies for size and/or shape-selective purification of nanoparticles. Current Opinion in Colloid \& Interface Science, 2011, 16(2): 135-148

25. Lee S A, Choo K H, Lee C H, Lee H I, Hyeon T, Choi W, Kwon H $\mathrm{H}$. Use of ultrafiltration membranes for the separation of $\mathrm{TiO}_{2}$ photocatalysts in drinking water treatment. Industrial \& Engineering Chemistry Research, 2001, 40(7): 1712-1719

26. Akthakul A, Hochbaum A I, Stellacci F, Mayes A M. Size fractionation of metal nanoparticles by membrane filtration. Advanced Materials, 2005, 17(5): 532-535

27. Wu M, Mao Z, Chen K, Bachman H, Chen Y, Rufo J, Ren L, Li P, Wang L, Huang T J. Acoustic separation of nanoparticles in continuous flow. Advanced Functional Materials, 2017, 27(14): 1606039

28. Barasinski M, Garnweitner G. Restricted and unrestricted migration mechanisms of silica nanoparticles in agarose gels and their utilization for the separation of binary mixtures. Journal of Physical Chemistry C, 2020, 124(9): 5157-5166

29. Konrath M, Brenner A K, Dillner E, Nirschl H. Centrifugal classification of ultrafine particles: influence of suspension properties and operating parameters on classification sharpness. Separation and Purification Technology, 2015, 156: 61-70

30. Winkler M, Sonner H, Gleiss M, Nirschl H. Fractionation of ultrafine particles: evaluation of separation efficiency by UV-vis spectroscopy. Chemical Engineering Science, 2020, 213: 115374

31. Kelland D R. Magnetic separation of nanoparticles. IEEE Transactions on Magnetics, 1998, 34(4): 2123-2125

32. Fraga García P, Brammen M, Wolf M, Reinlein S, Freiherr von Roman M, Berensmeier S. High-gradient magnetic separation for technical scale protein recovery using low cost magnetic nanoparticles. Separation and Purification Technology, 2015, 150: 29-36

33. Latham A H, Freitas R S, Schiffer P, Williams M E. Capillary magnetic field flow fractionation and analysis of magnetic nanoparticles. Analytical Chemistry, 2005, 77(15): 5055-5062

34. Carpino F, Zborowski M, Stephen Williams P. Quadrupole magnetic field-flow fractionation: a novel technique for the characterization of magnetic nanoparticles. Journal of Magnetism and Magnetic Materials, 2007, 311(1): 383-387

35. Wei G T, Liu F K. Separation of nanometer gold particles by size exclusion chromatography. Journal of Chromatography. A, 1999, 836(2): 253-260

36. Süß S, Metzger C, Damm C, Segets D, Peukert W. Quantitative evaluation of nanoparticle classification by size-exclusion chromatography. Powder Technology, 2018, 339: 264-272

37. Nomizu T, Nakashima H, Sato M, Tanaka T, Kawaguchi H. Magnetic chromatography of magnetic fine particles suspended in a liquid with a steel-bead column under a periodically intermittent magnetic field. Analytical Sciences, 1996, 12(6): 829-834

38. Kim S B, Nakada C, Murase S, Okada H, Ohara T. Development of magnetic chromatograph system for magnetic particle and ion separation with superconducting magnet. Physica C: Superconductivity and its Applications, 2007, 463-465: 1306-1310

39. Noguchi S, Kim S. Investigation on novel magnetic chromatography with ferromagnetic nano-wires for ion separation. IEEE Transactions on Applied Superconductivity, 2011, 21(3): 20682071

40. Arlt C R, Tschöpe A, Franzreb M. Size fractionation of magnetic nanoparticles by magnetic chromatography. Journal of Magnetism and Magnetic Materials, 2020, 497: 165967

41. Biehl P, von der Lühe M, Dutz S, Schacher F H. Synthesis, 
characterization, and applications of magnetic nanoparticles featuring polyzwitterionic coatings. Polymers, 2018, 10(1): 91

42. Satzer P, Wellhoefer M, Jungbauer A. Continuous separation of protein loaded nanoparticles by simulated moving bed chromatography. Journal of Chromatography. A, 2014, 1349: 44-49

43. Dyankova S, Doneva M, Todorov Y, Terziyska M. Determination of particle size distribution and analysis of a natural food supplement on pectin base. IOSR Journal of Pharmacy, 2016, 6(5): 1-8

44. Rajendran A, Paredes G, Mazzotti M. Simulated moving bed chromatography for the separation of enantiomers. Journal of
Chromatography. A, 2009, 1216(4): 709-738

45. Mazzotti M, Storti G, Morbidelli M. Supercritical fluid simulated moving bed chromatography. Journal of Chromatography. A, 1997, 786(2): 309-320

46. Lim J, Yeap S P, Che H X, Low S C. Characterization of magnetic nanoparticle by dynamic light scattering. Nanoscale Research Letters, 2013, 8(1): 381

47. Szepessy S, Thorwid P. Low energy consumption of high-speed centrifuges. Chemical Engineering \& Technology, 2018, 41(12): 2375-2384 